\title{
JOM Augmented Reality Mobile Shopping Behavior
}

\author{
Liew W. J. ${ }^{1}$ and JosephNg P. S. ${ }^{2}$ \\ ${ }^{1}$ Institute of Computer Science and Digital Innovation, UCSI University UCSI \\ Heights, 56000, Cheras, Kuala Lumpur, Malaysia \\ ${ }^{2}$ Faculty of Information Technology, Inti International University Persiaran Perdana \\ BBN Putra, 71800 Nilai, Negeri Sembilan, Malaysia \\ 1junwei190528@gmail.com, ${ }^{2}$ joseph.ng@newinti.edu.my
}

\begin{abstract}
The research aims to introduce Augmented Reality features into online shopping platforms to enhance consumer purchase behavior in online shopping platforms. Online shopping has become a common activity done by young people nowadays. More and more people choose to purchase online instead of buying at physical retail shops. This project has proposed to make online shopping item comparison more convenient and less time-consuming yet more visually for better-enhanced trust confidence. By providing augmented reality into online shopping platforms, the consumer can have a clear comparison between similar items, including price, product details, customer feedback, and review. This project is carried out in few private universities for data collection by using a mixed-method approach. The managerial and practical implication is discussed.
\end{abstract}

Keywords: Online shopping, Augmented reality, Consumer behavior, Purchase decision online payment security, Personal data privacy

\section{Introduction}

Based on the observation of recent trends, online shopping has become a phenomenon and growing rapidly. According to Lim Y. J. [1], $\$ 840$ billion of sales in 2014 from retail ecommerce has outdistanced the sales of US\$695 billion in the year 2013 and reach the highest point, which is US $\$ 1506$ billion in the year 2018. People have the desire to shop online because they can purchase everything without going out to physical retail shops [2]. People can compare the same products in different online shops virtually by viewing the feedback from the previous customers, check product details, and product sales per month [3].

Bringing the focus back to Malaysia, a Malaysia study indicated that there were around 26.69 million internet users in Malaysia in January 2020. This number of internet users is increased by $+3.6 \%$ compared to the year $2019.91 \%$ of these internet users within the aged 16 to 64 will search for a product online, and $90 \%$ of them will visit at least one online store to view the product details, and $82 \%$ of the users will purchase an item online after they visited the store for more information [4]. According to Malaysia Digital 2020 statistics [4], a total of sales in the fashion \& beauty category has reached $\$ 874.0$ million from the year 2019 to the year 2020 in Malaysia. Even though there are many research and statistics to point the online shopping is in rapid growth. However, there is a lack of research to show that AR does enhance the sales of online shopping platforms.

Article history:

Received (June 24, 2021), Review Result (July 30, 2021), Accepted (September 7, 2021) 
Augmented Reality (AR) is a computer-created object that provides an interface combining a real-life environment to a virtual environment by tracking an object body then apply a virtual effect on it [5]. According to Jozef's study [6] and Marzieh's study [7], Key factors that affect consumers' purchase behaviors are related to:

(a) Social proof

(b) Product details

(c) Product conditions

(d) Price

(e) Perceived risk

Consumers may not be able to visualize the effect of the products [6], like garments when buying online using traditional static non-AR features regret emotion is one of the extremely harmful emotions, and consumers will be afraid to purchase online at other times. From Sarwar's study [8], he concluded that regret is undeniable emotion, and online shopping will generate more regret than shop offline. Returning of broken products, long delivery time, and fairly affordable shipping fees are also impeding consumers to buy products online [9].

Table 1. Comparison of app features.

\begin{tabular}{|c|c|c|c|}
\hline Features & BuyBuy & Lazada & Shopee \\
\hline Product preview & $/$ & $/$ & $/$ \\
\hline Online shopping payment & $/$ & $/$ & / \\
\hline Location of the stores & $/$ & $\mathrm{X}$ & $\mathrm{X}$ \\
\hline Viewing item virtually & $/$ & $\mathrm{X}$ & $\mathrm{X}$ \\
\hline Compare item simultaneously & $/$ & $\mathrm{X}$ & $\mathrm{X}$ \\
\hline
\end{tabular}

Online shopping application feature is one of the factors that affect consumers' purchase behaviors. It brings a negative effect on consumers who have the intention to purchase the item when the applications took more time to load out the product's picture. Time-consuming, loading pictures from different online sellers may be time-consuming for consumers [10]. Online retailers are reluctant to use AR technology as the setup and operation may be costly. Lack of popularity of AR facilities in Malaysia, consumers may not be familiar with its function [11]. Privacy and securities, consumers may worry about the protection of their confidentiality, i.e., personal details [12].

\subsection{Literature review}

The release of Pokémon go does attract people's attention. This mobile application combines AR with virtual images. The idea of combining AR and games has generated tons of positive and negative effects in a short time. The Combination of AR with the game not only requires the players to walk around but also distracts the players' attention from the real environment [13].

According to Digi-Capital statistics [14], it predicts that AR market revenue will hit $\$ 90$ billion by 2020, and VR market revenue will remain unchanged at $\$ 30$ billion. Higher investment and innovation for AR and VR, surprisingly led by Asia and Asia (China, South Korea, Japan, others), could drive AR/VR revenue by 2020 [14].

Some of the Big companies such as IKEA, McDonald's, and more are developed their AR application to bring convenience to Customers. For example, IKEA's app allows users to see 
the effect of furniture display after purchased the furniture [15]. Thus, it increases user interaction with the brand and purchase intention. They can try the select products in homes by using a mobile application and a smartphone [16]. In 1984, the first idea of online shopping was started by TV shopping. In traditional offline shopping, consumers will need to go to the physical retails shop and have direct interaction with the product. In contrast, online shopping is restricted by the $2 \mathrm{D}$ screen to show the product's detail (size, color, actual quality), and the number of items present on the screen is limited [17]. However, consumers choose to purchase online is because they can buy their needs without going out to the physical shops. Fewer steps are taken to purchase an item and deliver it to the home [18].

People tend to buy items online because of the various selections, compare prices of similar items [19][20]. People do not need to go out and can grab the items they want through online shopping platforms and TV shopping. People can get a lower cost of the same item in different online shops [21]. People are not required to pay the process fees. An item from the process until reaches to consumer's hands is not an easy process. Item is produced through a layer-bylayer process from raw material until it becomes a useable product. Through this layer-by-layer process, the cost of product increment due to the taxes is charged [22].

Consumers do get uncomfortable when they are comparing items on one online shopping platform. Consumers have to swap out and click in another shop to view the items' detail. The loading time of product details might cause plenty of times [23]. People sometimes do not trust online shopping platforms. They are well aware that the seller might sell their privacy details to third-party companies [24]. Apart from that, they are afraid of item delivery failure and compensation risk, which may take plenty of time for the consumers [25].

\section{Problem statement, hypothesis}

By introducing AR features in the app, the users can view the virtual effect of items through the app. By doing so, the users can buy only the item they need. From the objectives delivered, all of the hypotheses will be declared in [Table 2].

Table 2. Research hypothesis

\begin{tabular}{|c|c|}
\hline H1 & Cost efficiency, AR can help consumers to save money on buying the right items. \\
\hline H2 & $\begin{array}{r}\text { Time efficiency, consumers can save time by using AR technology to view and } \\
\text { select the shopping items (clothes/garments). }\end{array}$ \\
\hline H3 & $\begin{array}{r}\text { Screening of step-by-step tutorial of using AR features for the first-time users has a } \\
\text { positive effect on encouraging the consumers to use the application. }\end{array}$ \\
\hline
\end{tabular}

H1: Cost efficiency, AR can help consumers to save money on buying the right items.

For instance, regret is one of the extreme emotions of consumers after they purchased the product. Especially for the first-timer, they will feel reluctant to repurchase if the first-time experience was not as their expectation. Therefore, consumers can make the right decision to buy the right products without regret.

H2: Time efficiency, consumers can save time by using AR technology to view and select the shopping items (clothes/garments).

Consumers will try to make a comparison between several similar items to purchase the right product. The page loading speed will lead consumers to lose their purchase intention towards the product. By viewing all items at a time will maintain the consumers' purchase intention and help them to compare the products easily. 
H3: Screening of step-by-step tutorial of using AR features for the first-time user has a positive effect on encouraging the consumers to use the application.

Implementation of AR may be complexed to certain consumers who are not digitally familiar. They may feel reluctant to try on this technology, whereby a tutorial video may help to clear their doubt.

\subsection{Value creations}

With all features provided in the app, users could virtually view the effect of garments. Thus, users can determine their minds to decide whether they want to spend their money on buying the items and reduce the time consumption on comparing the items in different online shops.

\section{Methodology}

This study will apply the mixed method to answer how and why questions from the interviewees through surveys and interviews. The data is to be collected via the following methodology, as summarized in the [Table 3] [29][30][31][32][33][34][35][36].

Table 3. Research methodology

\begin{tabular}{|c|c|}
\hline Research Dimension & Explanatory Sequential Design \\
\hline Research Methodology & Mixed Mode \\
\hline Research Methods & Survey \& Interview \\
\hline
\end{tabular}

The mixed-mode or mixed-method is applied by doing a random survey and interview as its primary data collection. To elaborate on the steps of data collection, the sequential design will illustrate, as shown in [Figure 1] [37][38][39][40][41][42].

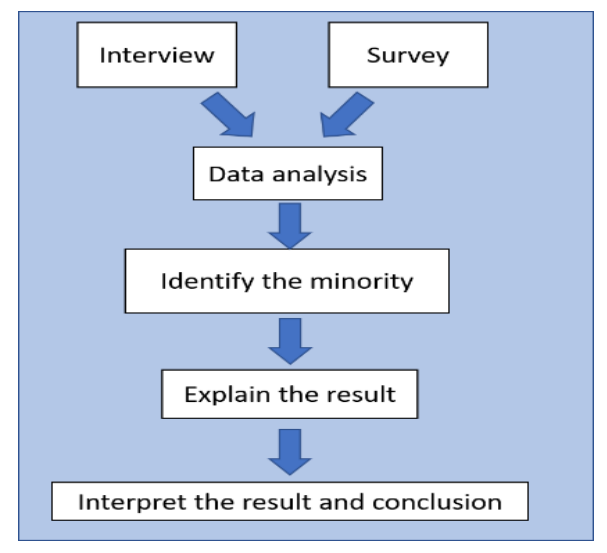

Figure 1. Sequential design

The online survey questionnaire and a short interview will be conducted at the same time by randomly picking respondents in private universities. After finishing the collection of data, general information will be gathered. Qualitative data is collected by questions the interviewees about the effect of AR on an online shopping platform. Finally, all the information will be interpreted and conclude together. Seven random respondents for the survey and four respondents for the interview are chosen to test whether the survey and interview questions are understandable. Several changes of survey and interview questions content and arrangement had been made to perform a better understanding of survey and interview questions. 


\subsection{Results and findings}

According to the survey research found, the majority of private university students are frequently using online shopping platforms to purchase items online. The candidates that took part in this survey are approximate $52.7 \%$ are male, and $47.3 \%$ are female.

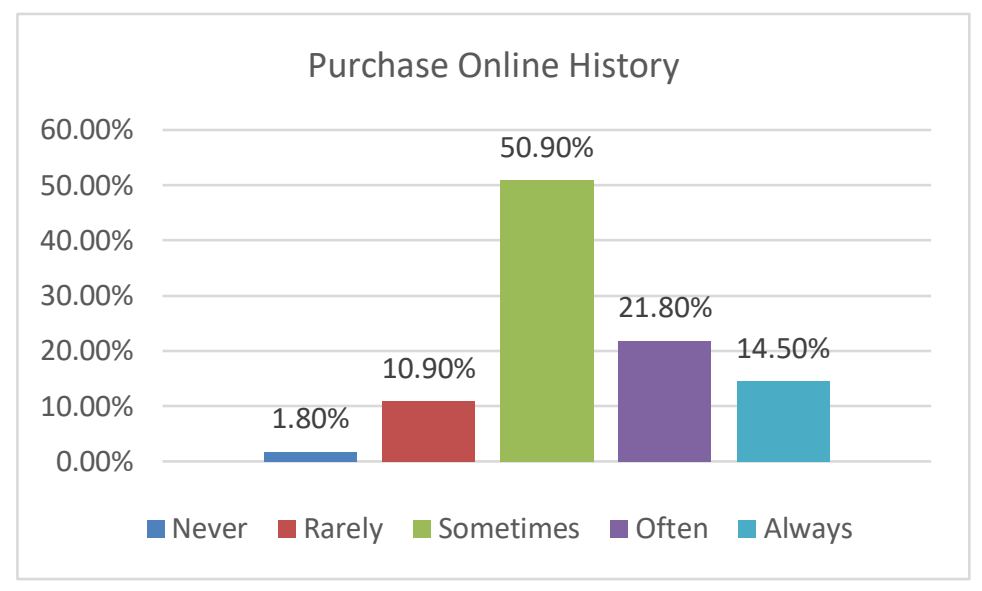

Figure 2. Purchase online history

[Figure 2] illustrated the respondents that have made at least one purchase online in the past 12 months. About 50\% of the respondents are focusing on the fashion and beauty category. Approximately $50 \%$ of the respondents said they were disappointed with their products buying online due to several factors, i.e., End up buying the thing that didn't need, the quality of cloth was not fit with the expectation, and the size of the garments are not accurate.

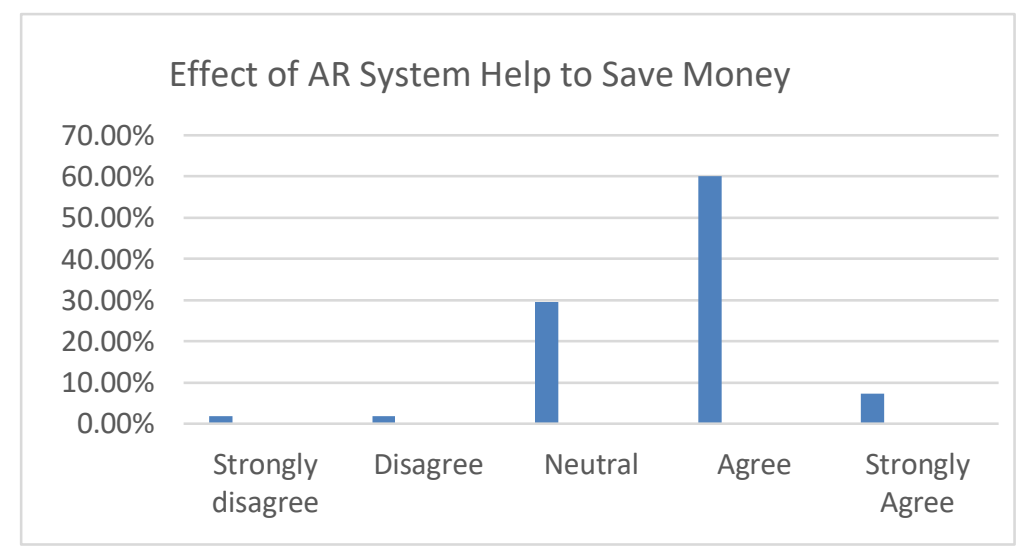

Figure 3. Effect of AR system help to save money

[Figure 3] shows that $67.3 \%$ of the respondents agreed that the AR system used in online shopping platforms could help them save money. This is because the AR system provides a computer-generated image for the consumers to view the virtual effect. Based on the result from the interview, the respondents agree that the AR system help to save money as they can determine whether the item is suitable or not. They will not spend their money on buying the wrong things, and there will be a higher chance to see the authenticity of the object and make the comparison. 
From the survey feedback form, $81.82 \%$ of respondents stated that they spent most of their time on online shopping platforms. The statement above does prove that the target group is fit with this research topic.

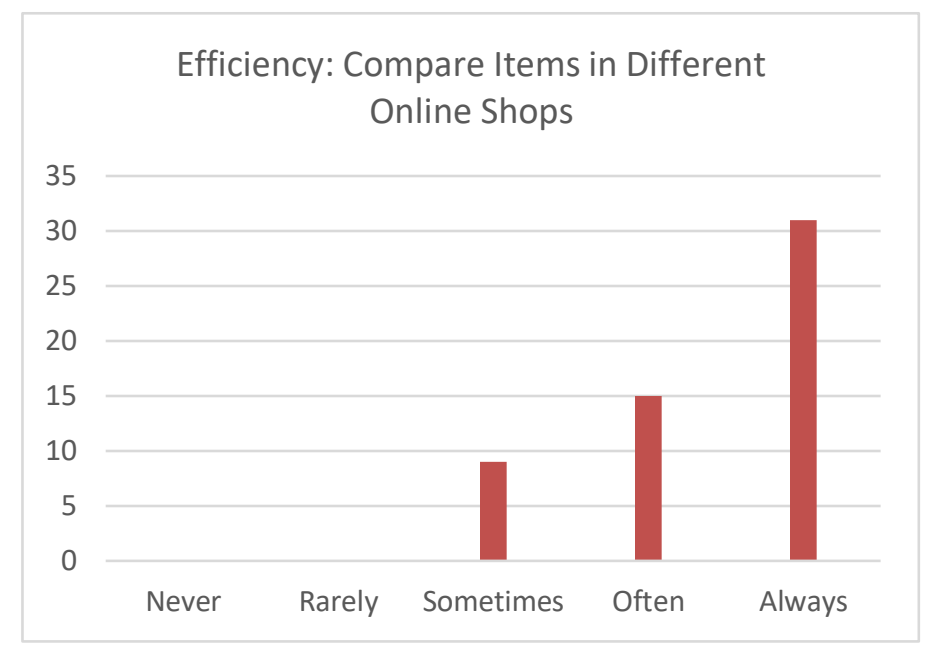

Figure 4. Respondents that were comparing the item in different online shops

[Figure 4] illustrates the candidates that were comparing different items on online shops. None of them will buy a product without comparing the thing in various online shops. This comparing can be in terms of product quality, price, shipping fees and duration, customer ratings, Etc. However, the majority of the candidates do not have relevant experience on an application that uses AR features, which means AR technology is not familiar in Malaysia.

Based on the interview feedback, candidates who do not agree with the efficiency of current online shopping are excellent. The reasons they told during the interview are because they have to switch between applications and shops to compare the similar items, which consume plenty of time to loading out the image and product details, difficult to have side by side comparison on current platforms, etc.

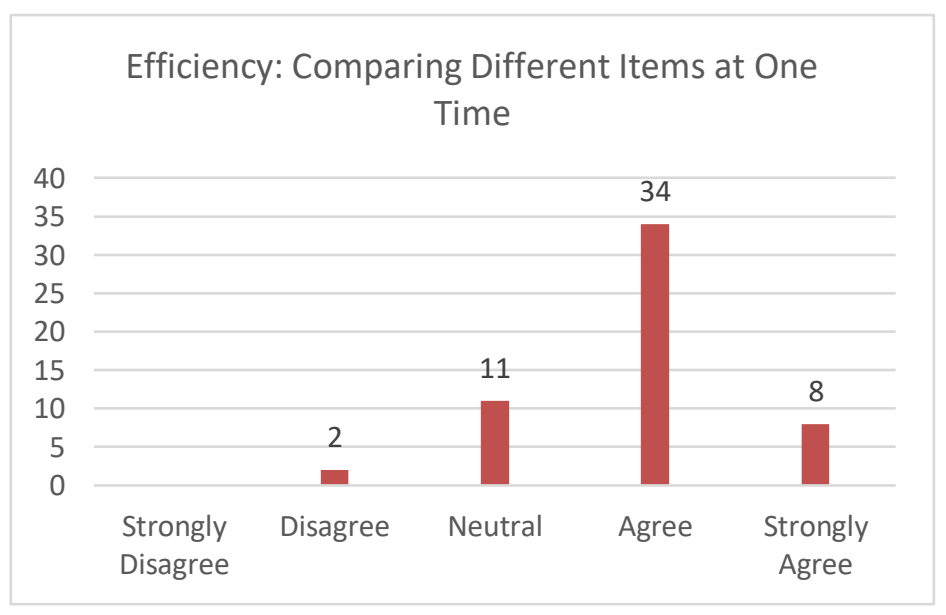

Figure 5. Comparing different items at one time 
According to [Figure 5], comparing different items at one time does enhance the efficiency of the online shopping platform. By viewing the product via AR does compare similar things at one time, which helps the consumers to save time. The respondents also agree with the statement. The AR system does aid in comparing items. Besides, this system helps consumers to know the product details, price, quality, and customer reviews. Consumers do not require to load and reload the items many times to compare stuff.

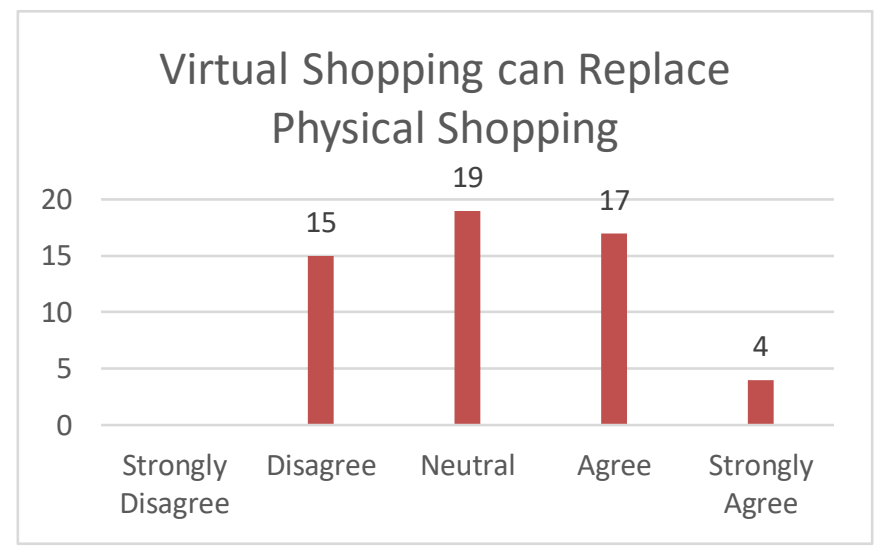

Figure 6. Virtual shopping can replace physical shopping

[Figure 6] illustrates that $38.2 \%$ of the respondents agree that virtual shopping with the AR system can replace physical shopping in the future, which is quite minor in the survey feedback. The candidates who are agreeing with the statement said, using the AR system in virtual shopping does make consumers easy to select the item they want to buy. Apart from that, it does save more time compared to shopping at a physical retails shop. In physical shops, no one can compare similar or the same item from different shops and prices, and so they will likely use an online shopping platform with AR features to buy the items they need. Besides, the respondents said buying items online can save their transportation costs, traffic jams, and avoid unnecessary exposure to the risk of viruses.

However, the respondents who disagree with this statement tell out differently. They would prefer buying stuff offline because they don't trust buying items online too much. As for real, most of the items sell online do not meet the quality, as the seller mentioned. They do not agree with the statement because physical shopping provides senses to feel, touch, see the item in reality, and also the experience and pleasure of physical shopping are always better.

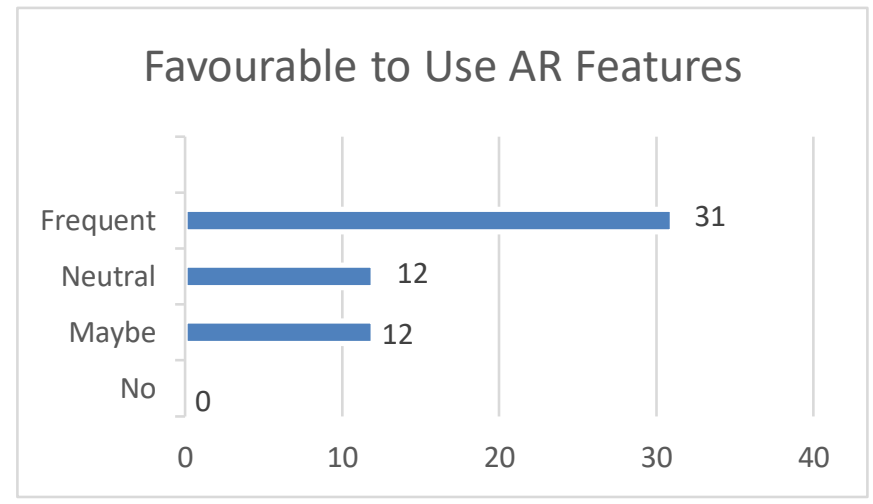

Figure 7. Favourable to use AR features 
As [Figure 7] shows, the majority of the respondents are willing to use the application with AR features. However, respondents doubt the complex operation of using AR features in online shopping platforms. During the interview section, the respondents are more willing to use the application when there is a step-by-step tutorial for first-time users.

\section{Conclusion}

In conclusion, the AR system is a new trend in online shopping platforms and has the potential to revolutionize mobile shopping by bringing products to life in front of the consumer through 3D models that consumers can size up and examine from all angles. This feature does help the consumers to save their cost spending on online shopping platforms. On the other hand, consumers can reduce time consumption while making the item comparison and swapping between several online shops. Based on the research finding, consumers require a step-by-step tutorial while first entered the application interface. This will help them understand how the software is working and what is the benefit of using this application. The limitation of this research paper is the coverage of the target group in this paper is only in two private universities in which the result might be only applicable within these university areas.

\subsection{Future works and limitations}

Future works maybe turn into implementation parts and investigate the image-quality control of the AR system. Due to several constraints (e.g., Network delay, Internet connection speed, hardware restriction) [30], the image might not wholly be loaded and result in a low-quality computer-generated image and users might not be able to view the effect. Therefore, network and hardware requirements and improvement might be interesting topics for further research.

\section{References}

[1] Y. J. Lim, A. Osman, S. N. Salahuddin, A. R. Romle, and S. Abdullah, "Factors influencing online shopping behavior: The mediating role of purchase intention," 7th International Economics and Business Management Conference, Kuantan, Malaysia, vol.35, pp.401-410, (2016)

[2] M. H. M. Javadi, H. R. Dolatabadi, M. Nourbakhsh, A. Poursaeedi, and A. R. Asadollahi, "An analysis of factors affecting the online shopping behavior of consumers," International Journal of Marketing Studies, vol.4, no.5, pp.81, (2012)

[3] N. Vasic, M. Kilibarda, and T. Kaurin, "The influence of online shopping determinants on customer satisfaction in the Serbian market," Journal of Theoretical and Applied Electronic Commerce Research, vol.14, no.2, pp.112. (2019)

[4] S. Kemp, "Digital 2020: Malaysia," DATAREPORTAL, Feb.18, 2020. Accessed on: Jun. 2, 2020. Available: https://datareportal.com/reports/digital-2020-malaysia

[5] W. J. Liew, P. S. Joseph Ng, H. C. Eaw, and K. Y. Phan, "JOM augmented reality effect towards online shopping platform," International Women in Engineering Conference on Electrical and Computer Engineering, Bhubaneswar, India, pp.29-32, (2020)

[6] J. R. Prasojo and P. S. Joseph Ng, "JomAR purchasing furniture in augmented reality experiences," Journal of Business Strategy and Automation, Forthcoming, (2021)

[7] I. Muktar, N. P. S. Joseph, P. Y. Choo, S. W. Wong, K. Y. Phan, and E. H. Lim, "Identifying mobile device requirements for a user interface in social networking," International Journal of Scientific Engineering and Technology, vol.3, no.10, pp.1230-1235, (2014)

[8] M. Loke, P. S. Joseph Ng, A. S. Shibghatullah, and H. C. Eaw, "JomDrone: Data mining financial sense in the property agency," Symposium on Industrial Electronics and Applications, Kuala Lumpur, Malaysia, pp.1-5, 
[9] C. Ng and C. Ramasamy, "Augmented reality marketing in Malaysia - Future scenarios,” Social Sciences, vol.7, no.11, pp.224-239, (2018)

[10] J. Bucko, L. Kakalej, and M. Ferencov, "Online shopping: Factors that affect consumer purchasing behavior," Cogent Business and Management, vol.5, no.1, pp.1-16, (2018)

[11] M. Zendehdel, L. Paim, and S. Osman, "Students online purchasing behavior in Malaysia: Understanding online shopping attitude," Cogent Business and Management, vol.2. no.1, pp.1-14, (2015)

[12] M. Sarwar, A. Awang, and M. Habib, "Consumer purchase regret: A systematic review," International Journal of Academic Research in Business and Social Sciences, vol.9, no.9, pp.403-425, (2019)

[13] M. S. Sohail, "Factors impeding online shopping: An Arab world perspective," European Online Journal of Natural and Social Sciences, vol.3, no.4, pp.972-982, (2014)

[14] C. Katawetawaraks and C. Wang, "Online shopper behavior: Influences of online shopping decision," Asian Journal of Business Research, vol.1, no.2, pp.66-74, (2011)

[15] N. Lazim and A. Khairul, "State-of-the-art responses on augmented reality application in Malaysia," International Journal on Sustainable Tropical Design Research and Practice, vol.8, no.2, pp.28-34, (2015)

[16] A. A. A. Basheer and A. A. Ibrahim, "Mobile marketing: Examining the impact of trust, privacy concern and consumers' attitudes on the intention to purchase. International journal of business and management," vol.5, no.3, pp.28-41, (2010)

[17] C. Ng and C. Ramasamy, “Augmented reality marketing in Malaysia - Future scenarios,” Social Sciences, vol.7, no.11, pp.224-239

[18] D. Capital, "Augmented/virtual reality revenue forecast revised to hit $\$ 120$ billion by 2020," Blog about Augmented, Virtual and Mixed Reality Industry | Digi-Capital. [online] Digi-capital.com. Available at: $<$ https://www.digi-capital.com/news/2016/01/augmentedvirtual-reality-revenue-forecast-revised-to-hit-120billion-by-2020/> [Accessed 3 June 2020]

[19] R. Paul, "IKEA catalog uses augmented reality to give a virtual preview of furniture in a room," [online] New Atlas. Available at: <https://newatlas.com/ikea-augmented-reality-catalog-app/28703/> [Accessed 29 May 2020]

[20] A. Tabusca, "Augmented reality-need, opportunity or fashion," Journal of Information Systems and Operations Management, vol.1, no.1, pp.1-10, (2014)

[21] Y. Lu and S. Smith, “Augmented reality e-commerce assistant system: trying while shopping,” In International Conference on Human-Computer Interaction, vol.10, no.2, pp.643-652, Springer, Berlin, Heidelberg, July, (2010)

[22] Y. C. Huang, S. Y. Hu, S. T. Wang, and S. C. C. Huang, "Exploration of virtual reality-based online shopping platform," In: Stephanidis C. (eds) HCI International 2019 - Posters. HCII 2019. Communications in Computer and Information Science, vol.1034, pp.81-88, Springer, Cham, (2019)

[23] R. Bansal, “Online shopping: A shining future,” International Journal of Techno-Management Research, vol.1, no.1, pp.1-10, (2013)

[24] S. L. Jarvenpaa and P. A. Todd, "Consumer reactions to electronic shopping on the world wide web," International Journal of Electronic Commerce, vol.1, pp.59-88, (1997)

[25] S. Lo, "Why expect lower prices online? Empirical examination in online and store-based retailers," International Journal of Electronic Commerce Studies, vol.5, no.1, pp.27-38, (2014)

[26] Corporate Finance Institute, "Product costs: Costs incurred in manufacturing a product, (2019) [online] Available at: https://corporatefinanceinstitute.com/resources/knowledge/accounting/product-costs/

[27] K. Zhao, "Research on consumer online purchasing decision and its influencing factors: Take Taobao as an example," Specialization in General Management, vol.1, no.1, pp.1-52, (2015)

[28] R. Saranya and M. Chandran, "Risks and threats in online shopping - A study with special reference to perception and behavior of customers in Chennai city," STAR Research Journal, vol.5, no.1, pp.1-11, (2017) 
[29] N. P. Joseph, A. K. Mahmood, P. Y. Choo, S. W. Wong, K. Y. Phan, and E. H. Lim, "IaaS cloud optimization during economic turbulence for Malaysia small and medium enterprise," International Journal of Business Information Systems, vol.16, no.2, pp.196-208, (2014)

[30] P. S. JosephNg, "EaaS optimization: Available yet hidden information technology infrastructure inside medium-size enterprise,” Technological Forecasting and Social Change, vol.132, pp.165-173, July, (2018)

[31] P. S. JosephNg, "EaaS infrastructure disruptor for MSE," International Journal of Business Information System, vol.30, no.3, pp.373-385, (2019)

[32] P. S. JosephNg and H. Eaw, "Making financial sense from EaaS for MSE during economic uncertainty," Future of Information and Communication Conference, Vancouver, Canada: Springer Advances in Intelligent Systems and Computing, pp.976-989, (2021)

[33] P. S. JosephNg and H. C. Eaw, "Economic turbulence and EaaS grid computing," Int. J. of Business Forecasting and Marketing Intelligence, forthcoming, (2021)

[34] P. S. JosephNg and H. C. Eaw, "Still technology acceptance model? Reborn with exon structure as a service," International Journal of Business Information Systems, forthcoming, (2021)

[35] P. S. JosephNg and C. M. Kang, "Beyond barebones cloud infrastructure services: Stumbling competitiveness during economic turbulence," Journal of Science and Technology, vol.24, no.1, pp.101-121, (2016)

[36] P. S, JosephNn, C. M. Kang, P. Y. Choo, S. W. Wong, K. Y. Phan, and E. Lim, "Exostructure services for infrastructure resources optimization," Journal of Telecommunication, Electronic, and Computer Engineering, vol.8, no.4, pp.65-69, (2016)

[37] N. S. Joseph, A. K. Mahmood, C. P. Yin, W. S. Wan, P. K. Yuen, and 1. E. Heng, "BareBone cloud IaaS: Revitalization disruptive technology," IEEE Symposium on Computer Applications and Industrial Electronics (ISCAIE), vol.18, no.1, pp.107-126, (2014)

[38] P. S. JosephNg, C. M. Kang, and K. Issa, "A study on integrating penetration testing into the information security framework for Malaysian higher education institutions," Malaysia: International Symposium on Mathematical Sciences and Computing Research (iSMSC), (2015)

[39] P. S. JosephNg, C. M. Kang, P. Y. Choo, S. W. Wong, K. Y. Phan, and E. H. Lim, "Beyond cloud infrastructure services in medium-size manufacturing," International Symposium on Mathematical Sciences and Computing Research (iSMSC), Ipoh: IEEE, pp.150-155, (2015)

[40] P. S. JosephNg, C. M. Kang, A. K. Mahmood, S. W. Wong, K. Y. Phan, S. H. Saw, and J. T. Lim, "EaaS: Available yet hidden infrastructure inside MSE," Proceedings of the Fifth International Conference on Network, Communication, and Computing, Kyoto, pp.17-21, (2016)

[41] P. S. JosephNg, Y. F. Loh, and H. C. Eaw, "Grid computing for MSE during volatile economy," 20th International Conference on Control, Automation, and Systems (ICCAS), Busan, Korea: IEEE, pp.709-714, (2020)

[42] J. Ng-Poh-Soon, C. P. Yin, W. S. Wan, and. S. Nazmudeen, "Energizing ICT infrastructure for Malaysia SME competitiveness during economic turbulence," Student Conference on Research and Development, pp.310-314, Cyberjaya, Malaysia: IEEE

[43] P. Clini, E. Frontoni, R. Quattrini, and R. Pierdicca, “Augmented reality experience: From high-resolution acquisition to real-time augmented contents," Advances in Multimedia, pp.1-9, (2014), DOI: $10.1155 / 2014 / 597476$ 